Int. J. Morphol.,

31(3):911-914, 2013.

\title{
Irrigación Renal: Multiplicidad de Arterias
}

\author{
Renal Irrigation: Multiplicity of Arteries
}

Cruzat, C.* \& Olave, E.**

CRUZAT, C. \& OLAVE, E. Irrigación renal: Multiplicidad de arterias. Int. J. Morphol., 31(3):911-914, 2013.

RESUMEN: Generalmente, el riñón consta de una arteria renal originada en la aorta abdominal, que luego se divide en una rama anterior y posterior, sin embargo no es raro encontrar más de una emergiendo de la parte abdominal de la aorta o de alguna arteria ilíaca. El incremento en el uso de la nefrectomía laparoscópica ha llevado a la necesidad de un conocimiento detallado de la anatomía vascular renal. Por la importancia de las variaciones arteriales renales, se presenta una disposición de irrigación múltiple, que fue observada en un individuo adulto de 54 años de edad y de sexo masculino, a través de una tomografía computarizada multidetectores, con reconstrucción tridimensional. En el lado derecho, se observaron cuatro arterias renales (AR), con origen independiente desde la parte abdominal de la aorta, donde la AR1 y AR4 ingresan en la parte inferior del seno renal y la AR2 y AR3 en la parte superior del mismo. En el lado izquierdo, se observaron dos arterias renales, donde la más superior (AR1) es la de mayor calibre. Casos con más de tres arterias son raros, de tal modo que la variación presentada en este artículo es de interés anatómico y clínico, por la presencia de cuatro arterias en el lado derecho y de dos en el lado izquierdo en un mismo individuo.

PALABRAS CLAVE: Anatomía; Riñón; Arterias.

\section{INTRODUCCIÓN}

Con el continuo avance de la tecnología de imágenes, se ha podido observar en individuos vivos una gran variedad en la disposición de diversas estructuras anatómicas, particularmente en el sistema vascular. Durante mucho tiempo, la disección mostró los padrones de irrigación de los diferentes órganos, pero las imágenes permiten ver directamente en vivos a estos vasos, pudiendo realizar una planificación correcta para diversos procedimientos quirúrgicos que se tuvieran que realizar en dichos órganos.

Como el desarrollo del sistema urogenital es más complejo que otros sistemas del cuerpo, existe una mayor presencia de anomalías congénitas respecto al resto de los sistemas, siendo las del riñón y sus vasos relativamente comunes. Pocas causan sintomatología clínica, sin embargo pueden predisponer a patologías renales (Krishnaveni \& Roopa Kulkarni, 2013).

Generalmente, el riñón consta de una arteria renal originada en la aorta abdominal, que luego se divide en una rama anterior y posterior, sin embargo no es raro encontrar más de una emergiendo de la parte abdominal de la aorta o de alguna arteria ilíaca (Olave et al., 2007; Raheem et al.,
2008; Krishnaveni \& Roopa Kulkarni) o en raras ocasiones, pudiendo originarse en la parte baja de la aorta torácica o arterias mesentéricas (Urban et al., 2001). El incremento en el uso de la nefrectomía laparoscópica ha llevado a la necesidad de una evaluación imagenológica detallada de la anatomía vascular renal (Kumar et al., 2010).

Este artículo muestra la presencia de cuatro arterias destinadas al riñón derecho y dos al riñón izquierdo de un mismo individuo y analiza la multiplicidad de arterias descritas por diversos autores.

\section{MATERIAL Y MÉTODO}

Las variaciones de la disposición arterial renal, presentada en este artículo, fueron observadas en un individuo adulto de 54 años de edad y de sexo masculino, a través de una tomografía computarizada multidetectores, con reconstrucción tridimensional. En las imágenes obtenidas se analizaron y describieron las diversas arterias que eran destinadas a los riñones. 


\section{DESCRIPCIÓN}

En el lado derecho, se observaron cuatro arterias renales (Figs. 1 y 2), las dos más superiores (AR1 y AR2) con origen independiente desde la parte abdominal de la aorta, distanciada una de la otra y las dos inferiores (AR3 y AR4) con origen casi en el mismo punto de la aorta, siendo ligeramente superior el origen de la penúltima. De arriba abajo, la AR1 y la AR3 son de calibre semejante, siendo un poco más calibrosa la AR2. La AR4 es la de mayor calibre. La AR1 y AR4 ingresan en la parte inferior del seno renal y la AR2 y AR3 en la parte superior del mismo.

En el lado izquierdo (Figs. 1 y 3), se observaron dos arterias renales, donde la más superior (AR1), de mayor calibre, a poco de su origen en la aorta, a nivel de la AR2 del lado derecho, emite una rama (R1) que se dirige a la parte superior del riñón, para luego dividirse en dos ramas, superior e inferior, donde la primera ingresa en la parte superior del seno renal y la segunda, en la parte inferior del mismo. $\mathrm{La}$ arteria renal inferior (AR2) se origina en la aorta, a nivel del origen de AR4 en el lado derecho, para luego dividirse en dos ramas, a nivel del nacimiento de la R1 de AR1, donde la rama más superior, de menor calibre que la inferior, ingresa en el seno renal, por debajo del ingreso de la rama superior de AR1. La rama inferior, realizando un trayecto bajo la rama inferior de AR1, la cruza posteriormente antes de ingresar en el seno renal por encima de ésta.

\section{DISCUSION}

La irrigación renal está dada generalmente por arterias que se originan directamente de la aorta, una de cada lado, siendo la derecha de mayor longitud que la izquierda, arterias que se dividen antes de su ingreso en el seno renal, en dos o más ramas (Testut \& Latarjet, 1969; Williams et al., 1995; Moore \& Dalley, 2002).

De acuerdo al conocimiento embriológico, entre la sexta y novena semana, los riñones ascienden de su posición originalmente sacra a una posición lumbar y se sitúan inmediatamente por debajo de las glándulas suprarrenales, siguiendo su trayecto justo a cada lado de la aorta dorsal. El mecanismo de este ascenso se desconoce, aunque en él podría intervenir el crecimiento diferencial de las regiones lumbar y sacra del embrión. A medida que este órgano asciende, va siendo vascularizado por distintos brotes arteriales procedentes de la aorta dorsal, con desaparición de la arteria renal original de la región sacra (Larsen, 2003)

Las variaciones en este proceso de ascensión pueden provocar distintas anomalías. En casos raros, un riñón no asciende en absoluto, permaneciendo como riñón pélvico (Rodrigues et al.,1997; Larsen). A veces, una o varias de las arterias renales inferiores transitorias no regresan, sino que persisten como arterias renales acceso-

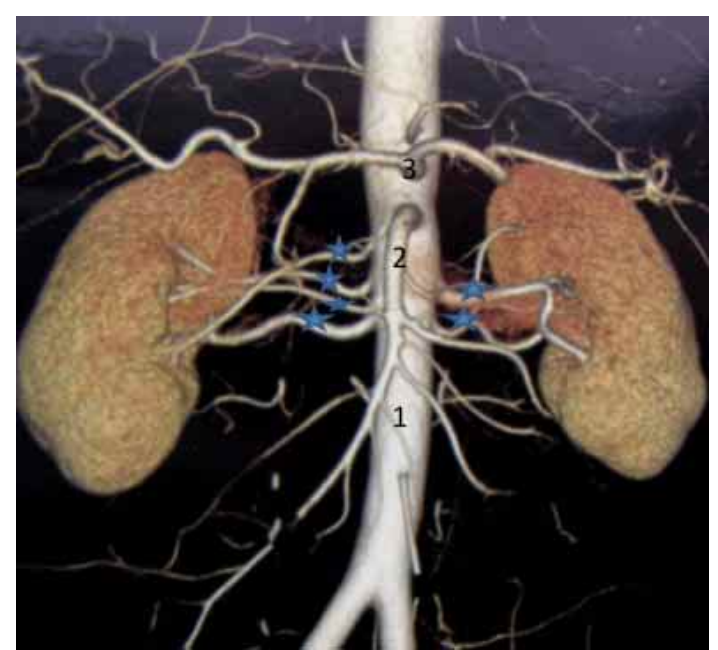

Fig. 1. Visión anterior de la parte abdominal de la aorta y sus ramas. Se observan cuatro arterias renales en el lado derecho y dos en el izquierdo (estrellas).

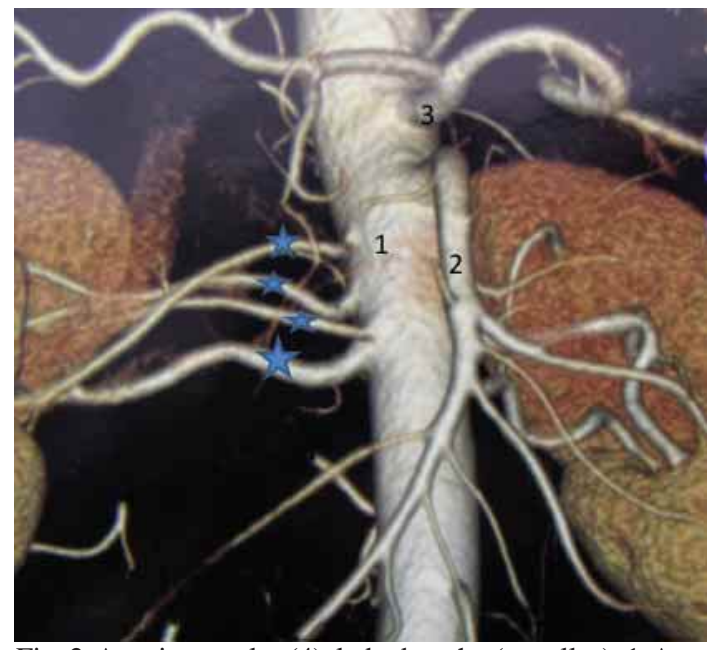

Fig. 2. Arterias renales (4), lado derecho (estrellas); 1. Aorta, 2. Arteria mesentérica superior, 3. Tronco celíaco.

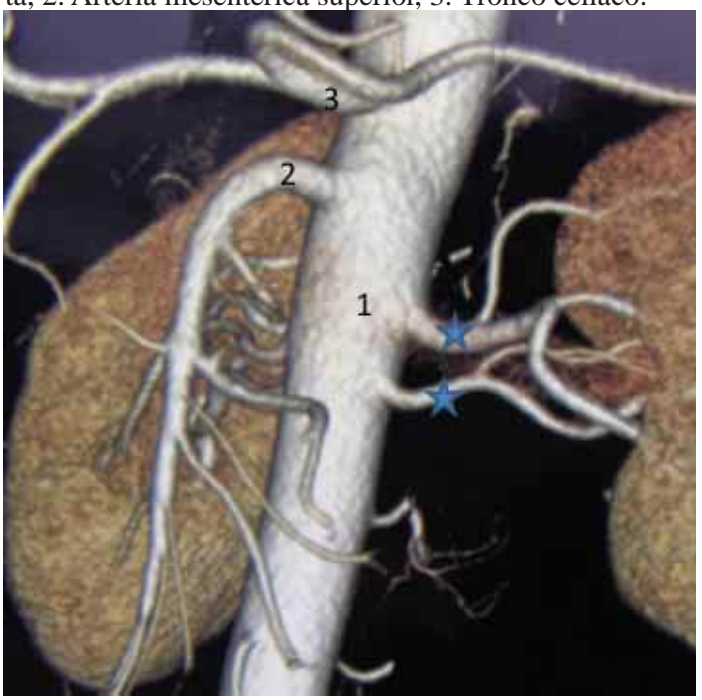

Fig. 3. Arterias renales (2), lado izquierdo (estrellas); 1. Aorta, 2. Arteria mesentérica superior, 3. Tronco celíaco. 
rias. Los polos inferiores de los dos mesonefros pueden fusionarse durante el ascenso, formando un riñón en herradura con forma de U, que cruza la cara ventral de la aorta. Durante la ascensión, este riñón queda atrapado bajo la arteria mesentérica inferior, por lo que no alcanza su localización normal (Larsen).

Casos de multiplicidad de arterias han sido publicados por varios autores y en porcentajes variables. Así, Moore (1984) describió que un 25\% de los individuos podría tener más de una arteria renal; Wozniak (2000) señaló esta variación en $11,2 \%$; en 2004, Khamanarong et al. y Dhar \& Lal en 2005, informaron un $18 \%$ y un $20 \%$, respectivamente. Por su parte, Oskan et al. (2006) estudiaron 855 pacientes observando esta variación en $24 \%$ de los casos. Otros autores han presentado casos aislados de individuos con más de una arteria renal (Bulic et al., 1996; Loukas et al., 2005; Tanyeli et al., 2006; Deepthinath, 2006, Olave et al.; Panagouli et al., 2012, entre otros).

Casos con más de tres arterias son raros, de tal modo que la variación presentada en este artículo es de interés anatómico y clínico, por la presencia de cuatro arterias en el lado derecho y de dos en el lado izquierdo en un mismo individuo, teniendo en cuenta que las arterias múltiples son encontradas entre un 18 y $30 \%$ unilateralmente y en $10 \%$ bilateralmente (Desai et al., 2007). Recientemente, Krishnaveni \& Roopa Kulkarni, describieron un caso que presentaba cinco arterias renales en el lado derecho (una principal y cuatro accesorias), inclusive la más inferior originándose desde la arteria ilíaca común derecha. En el lado izquierdo, se presentaron dos arterias renales. El riñón derecho estaba en una posición más baja que el del lado izquierdo, teniendo su polo superior a nivel del disco intervertebral entre las vértebras L1 y L2.

La mayoría de los cirujanos de trasplantes renales prefieren los riñones izquierdos con una única arteria renal, debido a la mayor longitud de la vena renal comparado con la vena renal derecha. Sin embargo, no todos los potenciales donadores tienen una anatomía vascular convencional. Múltiples arterias renales son encontradas entre 18 y $43 \%$ de los potenciales donadores vivos y es por esta razón que el uso de riñones con múltiples arterias es necesario ya que los potenciales beneficios de la donación pueden ser ofrecidas a un mayor número de candidatos a trasplante (Chedid et al., 2013).

Con respecto a los trasplantes de riñones con múltiples arterias o con arterias únicas, diversos estudios han comparado estos riñones y no se han observado en los pacientes trasplantados diferencias significativas en complicaciones vasculares o urológicas, de tal modo que se ha indicado que trasplantar uno con varias arterias es igualmente seguro que aquellos que tienen una sola arteria (Chedid et al.; Ashraf et al., 2013).

Como se ha señalado, el conocimiento de las variaciones en la irrigación renal es muy importante y las imágenes obtenidas con equipos muy desarrollados técnicamente han permitido conocer con detalles la disposición del árbol arterial, lo que lleva entonces, a una mejor planificación de la cirugía del trasplante renal.

CRUZAT, C. \& OLAVE, E. Irrigación renal: Multiplicidad de arterias. Int. J. Morphol., 31(3):911-914, 2013.

SUMMARY: Generally, the kidney has a one renal artery originating from the abdominal aorta, which is then divided into an anterior and posterior branches, however not uncommon to find more than one emerging from the abdominal aorta or iliac artery some. The increased use of laparoscopic nephrectomy has led to the need for detailed knowledge of renal vascular anatomy. Given the importance of the renal artery variations, we present a multiple irrigation of the kidney, which was observed in an adult man of 54 years old, through multidetector computed tomography with three-dimensional reconstruction. On the right side, there were four renal arteries (RA), with an independent origin from the abdominal aorta, where RA1 and RA4 entering the bottom of the renal sinus and RA3 and RA2 at the superior part of this sinus. On the left, we observed two renal arteries where the most superior (RA1) is the largest size. Cases with more of three arteries are rare, so that the variation presented in this article had anatomical and clinical interest for the presence of four arteries on the right side and two on the left side in an individual.

KEY WORDS: Anatomy; Kidney; Arteries.

\section{REFERENCIAS BIBLIOGRÁFICAS}

Ashraf, H.; Hussain, I.; Siddiqui, A. A.; Ibrahim, M. N.; \& Khan, M.U. The outcome of living related kidney transplantation with multiple renal arteries. Saudi J. Kidney Dis. Transpl., 24(3):615-619, 2013.
Bulic, K.; Ivkic, G. \& Pavic T. A case of duplicated right renal artery and triplicated left renal artery. Ann. Anat., 178(3):2813, 1996. 
Chedid, M.; Muthu, C.; Nyberg, S.; Lesnick, T.; Kremer, W.; Prieto, M.; Heimbach, J.Chow, G.; Stegall, M. \& Dean, P. Living donor kidney transplantation using laparoscopically procured multiple renal artery kidneys and right kidneys. J. Am. Coll. Surg., 217(1):144-52, 2013.

Deepthinath, R.; Satheesha Nayak, B.; Mehta. R. B.; Bhat, S.; Rodriguez, V.; Samuel, V. P.; Venkataramana, V. \& Prasad AM. Multiple variations in the paired arteries of the abdominal arteries. Clin Anat., 19(6):566-8, 2006.

Desai, M. R.; Ganpule, A. P.; Gupta, R. \& Thimmegowda, M. Outcome of renal transplantation with multiple versus single renal arteries after laparoscopic live donor nephrectomy: a comparative study. Urology, 69(5):824-7, 2007.

Dhar, P. \& Lal, K. Main and accessory renal arteries-a morphological study. Ital. J. Anat. Embryol., 110(2):101-10, 2005.

Khamanarong, K; Prachaney, P.; Utraravichien, A; Tong-Un, T. \& Sripaoraya, K. Anatomy of renal arterial supply. Clin. Anat., 17(4):334-6, 2004.

Krishnaveni, C. \& Roopa Kulkarni. A right ectopic kidney with bilateral multiple anomalies of the renal vasculature - A case report. J.Clin.Diag.Res., 7(1):150-153, 2013.

Kumar, S.; Neyaz, Z. \& Gupta, A. The utility of 64 channel multidetector CT angiography for evaluating the renal vascular anatomy and possible variations: a pictorial essay. Korean $J$. Radiol., 11(3):346-54, 2010.

Larsen, W. Embriología Humana. $3^{\text {a }}$ Ed. Madrid, Elsevier, 2003.

Loukas, M.; Aparicio, S.; Beck, A.; Calderon, R. \& Kennedy, M. Rare case of right accessory renal artery originating as a common trunk with the inferior mesenteric artery: a case report. Clin. Anat., 18(7):530-5, 2005.

Moore, K. L. \& Dalley, A. F. Anatomia con Orientación Clinica. $4^{a}$ Ed. Buenos Aires, Panamericana, 2002.

Olave, E.; Henríquez, J.; Puelma, F.; Cruzat, C. \& Soto, A. Arterias renales mútiples. Int. J. Morphol., 25(4):927-930, 2007.

Oskan, U.; Oguskurt, L.; Tercan, F.; Kizilkikiliç, O.; Koç, Z. \& Koca, N. Renal artery origins and variationes: angiographic evaluation of 855 consecutive patients. Diagn. Interv. Radiol., $12: 183-6,2006$.

Panagouli, E.; Lolis, E. \& Venieratos, D. Bilateral origin of the testicular arteries from the lower polar accessory renal arteries. Int.J. Morphol., 30(4):1316-20, 2012.

Raheem, O.; Glacken, P.; O’Brien, M.; Hickey, D. \& Mohan, P. A single male cadaver with multiple renal arteries. Ir. J. Med. Sci., 177:265-7, 2008.
Rodrigues, C. F. S.; Olave, E.; Gabrielli, C. \& Sousa, L. M. C. Consideraciones anatómicas sobre la fusión renal. Relato de un caso. Rev. Chil. Anat., 15(1):51-5, 1997.

Tanyeli, E.; Uzel, M. \& Soyluoglu, A.I. Complex renal vascular variation: a case report. Ann. Anat., 188(5):455-8, 2006.

Testut, L. \& Latarjet, A. Tratado de Anatomia Humana. Barcelona, Salvat, 1969.

Urban, B.A.; Ratner, L.E. \& Fishman, E.K. Three-dimensional volume-rendered CT angiography of the renal arteries and veins:normal anatomy, variants and clinical applications. Radiographics, 21:373-86, 2001.

Wozniak, W. T. Origin of the renal arteries from sides of aorta. Folia Morphol (Warsz)., 58(4):259-61, 2000.

Williams, P. L.; Warwick, R.; Dyson, M. \& Bannister, L. H. Gray Anatomia. 37 ed. Rio de Janeiro, Guanabara-Koogan, 1995.

Dirección para correspondencia:

Prof. Dr. Enrique Olave Riffo

Facultad de Medicina

Universidad de La Frontera

Casilla 54-D

Temuco

CHILE

Email: enrique.olave@ufrontera.cl

Recibido : 18-02-2013

Aceptado: 27-04-2013 